\title{
Obstructive sleep apnea and associated factors among hypertensive patients attending a tertiary cardiac center in Tanzania: a comparative cross-sectional study
}

Pedro Pallangyo ${ }^{1,2^{*}}$ D, Lucy R. Mgopa ${ }^{3}$, Zabella Mkojera ${ }^{1}$, Makrina Komba ${ }^{1}$, Jalack Millinga ${ }^{4}$, Nsajigwa Misidai ${ }^{1}$, Happiness J. Swai ${ }^{1}$, Henry Mayala ${ }^{5}$, Smita Bhalia ${ }^{2}$, Salma Wibonela ${ }^{4}$ and Mohamed Janabi

\begin{abstract}
Background: There is mounting evidence for a reciprocal yet bidirectional association between sleep-disordered breathing and hypertension. Obstructive sleep apnea (OSA), a common cause of systemic hypertension is an independent risk factor for hypertension-related cardiovascular morbidity and mortality. In this comparative hospitalbased cross-sectional study, we sought to explore the burden of obstructive sleep apnea and its associated risk factors among hypertensive patients attending Jakaya Kikwete Cardiac Institute.

Methodology: A total of 1974 individuals (i.e. 1289 hypertensive and 685 normotensives) were consecutively enrolled in this study. The Berlin questionnaire and Epworth Sleepiness Scale were utilized in the assessment of OSA and excessive daytime sleepiness (EDS) respectively. Logistic regression analyses were employed in the determination of associated factors for OSA.

Results: The mean age was 53.4years and females constituted the large majority (60.4\%) of participants. About three quarters (74.1\%) of participants had excess body weight, $11.6 \%$ had diabetes, $8.0 \%$ had asthma and $18.6 \%$ had history of recurrent nasal congestion. Positive family history of snoring was reported by $43.1 \%$ of participants and $36.9 \%$ had a personal history of snoring. Persons with hypertension displayed a higher frequency (42.1\%) of OSA compared to their normotensive counterparts (11.8\%), $p<0.001$. Multivariate logistic regression analyses revealed hypertension (OR $5.1,95 \% \mathrm{Cl} 3.2-8.2, p<0.001$ ), diabetes mellitus (OR 2.2, 95\% Cl 1.3-3.5, $p<0.01$ ), chronic nasal congestion (OR 1.6, 95\% $\mathrm{Cl} 1.1-2.5, p=0.01$ ), obesity (OR $2.4,95 \% \mathrm{Cl} 1.8-3.3, p<0.001)$, increased neck circumference (OR 2.7, 95\% Cl 1.2-6.4, $p=0.02$ ), family history of snoring (OR 5.5, 95\% Cl 4.0-7.5, $p<0.001)$, and working $>8 \mathrm{~h} / 24 \mathrm{~h}(\mathrm{OR} 0.6,95 \% \mathrm{Cl} 0.4-1.0$, $p=0.03$ ) to have an independent association for OSA. Furthermore, participants with hypertension displayed superior odds for OSA compared to their normotensive counterparts across all subgroup analyses.
\end{abstract}

Conclusion: OSA is considerably common among patients with hypertension in a tertiary health care setting in Tanzania. Positive family history of snoring was the strongest associated factor; however, excess body weight proved to be the strongest modifiable risk factor. In view of its pervasiveness, OSA should be an integral part of the medical evaluation in hypertensive individuals.

\footnotetext{
${ }^{*}$ Correspondence: pedro.pallangyo@gmail.com

${ }^{2}$ Cardiology, Jakaya Kikwete Cardiac Institute, P.O Box 65141, Dar es Salaam, Tanzania

Full list of author information is available at the end of the article
}

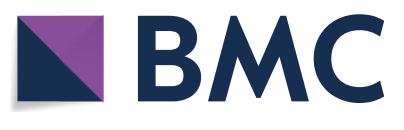

(c) The Author(s) 2021. Open Access This article is licensed under a Creative Commons Attribution 4.0 International License, which permits use, sharing, adaptation, distribution and reproduction in any medium or format, as long as you give appropriate credit to the original author(s) and the source, provide a link to the Creative Commons licence, and indicate if changes were made. The images or other third party material in this article are included in the article's Creative Commons licence, unless indicated otherwise in a credit line to the material. If material is not included in the article's Creative Commons licence and your intended use is not permitted by statutory regulation or exceeds the permitted use, you will need to obtain permission directly from the copyright holder. To view a copy of this licence, visit http://creativecommons.org/licenses/by/4.0/. 
Keywords: OSA, Sleep-related breathing disorders, Sleep apnea, Obstructive sleep apnea, Snoring, Hypertension, Elevated blood pressure, High blood pressure, Developing world, Sub Saharan Africa, Dar Es Salaam, Tanzania

\section{Background}

There is mounting evidence for a reciprocal yet bidirectional, and a complex however intriguing association between sleep-disordered breathing and hypertension (Kario 2009; Lavie et al. 2000; Calhoun and Harding 2010; Börgel et al. 2004; Logan et al. 2001; Peppard et al. 2000a; Lavie and Hoffstein 2001; Bixler et al. 2000; Young et al. 1997a; Nieto et al. 2000; Marin et al. 2012; Pedrosa et al. 2011; Gonçalves et al. 2007; Hou et al. 2018; SapiñaBeltrán et al. 2019; Patel et al. 2019; Ahmad et al. 2017; Belyavskiy et al. 2019; Tam et al. 2019). Obstructive sleep apnea (OSA), a common cause of systemic hypertension is associated with poor blood pressure control and resistant hypertension (Kario 2009; Lavie et al. 2000; Calhoun and Harding 2010; Börgel et al. 2004; Logan et al. 2001; Peppard et al. 2000a; Lavie and Hoffstein 2001; Bixler et al. 2000; Young et al. 1997a; Nieto et al. 2000; Marin et al. 2012; Pedrosa et al. 2011; Gonçalves et al. 2007; Hou et al. 2018; Sapiña-Beltrán et al. 2019; Patel et al. 2019; Ahmad et al. 2017; Belyavskiy et al. 2019; Tam et al. 2019). The mechanisms underlying the association between OSA and hypertension have not been well elucidated. Nevertheless, sympathetic nervous system overactivity is considered to be the primary mechanism linking OSA to the development of hypertension. It has been postulated that the intermittent hypoxia (i.e. hypoxemic - apneic episodes) induced by OSA leads to chemoreceptor activation and increased sympathetic outflow causing inflammation, neurohormonal dysregulation and endothelial dysfunction that predisposes to increased carotid intima-media thickness and arterial stiffness inevitably leading to the development of hypertension (Steiropoulos et al. 2010; Zhang and Si 2012; Wolf and Narkiewicz 2017; Jelic et al. 2002; Minoguchi et al. 2005; Jehan et al. 2020; Fava et al. 2014).

Although less than one-tenth of the general adult population is estimated to be affected (Epstein et al. 2009), OSA is known to distress between 30 and $80 \%$ of hypertensives and $70-90 \%$ of the resistant hypertension subgroup (Epstein et al. 2009; Parati et al. 2014; Parati et al. 2012; Somers et al. 2008). Conversely, between 35 and $80 \%$ of individuals with OSA have elevated blood pressure (Parati et al. 2012; Worsnop et al. 1998; DuránCantolla et al. 2009; Parati et al. 2013). Evidence from epidemiological studies strongly implicate OSA as an independent risk factor for hypertension-related cardiovascular morbidity (i.e. arrhythmias, cerebrovascular disease, ischemic heart disease, large vessel disease and heart failure) (Somers et al. 2008; Leung and Bradley 2001; Pinto et al. 2016; Gottlieb et al. 2010; Yaggi et al. 2005; Redline et al. 2010; Shahar et al. 2001; Lattimore et al. 2003; Peker et al. 1999; Mooe et al. 2000; Shah et al. 2010) and mortality (Yaggi et al. 2005; Young et al. 2008; Marshall et al. 2008; Punjabi et al. 2009). Additionally, OSA is associated with increased incidence of numerous nocturnal cardiovascular events including angina pectoris, myocardial infarction and sudden cardiac death (Gami et al. 2005; Kuniyoshi et al. 2008). Owing to the paucity of data on the association between OSA and hypertension in sub- Saharan Africa (SSA) in general and Tanzania in particular, we conducted this comparative hospital-based cross-sectional study to explore the burden of OSA and its associated factors among hypertensive patients attending Jakaya Kikwete Cardiac Institute (JKCI).

\section{Methods}

Study design, recruitment process, and definition of terms This comparative cross-sectional hospitalized-based study was conducted at JKCI, a tertiary care public teaching hospital in Dar es Salaam, Tanzania between July 2020 and March 2021. A consecutive sampling method was utilized to recruit consented hypertensive outpatients (cases) and normotensive patient escorts (controls) during their scheduled clinic visit. A structured questionnaire bearing questions pertaining to sociodemographic and clinical characteristics, measurement of key vitals (blood pressure, blood sugar, height, weight, and waist and neck circumference) was used during participants' interviews. Moreover, standard tools (i.e. the Berlin questionnaire (Tan et al. 2017) and Epworth sleepiness scale (Drakatos et al. 2015)) were utilized in the assessment of OSA and EDS respectively. According to a study by Tan $A$ and colleagues, the specificity, sensitivity, positive and negative predictive value of the Berlin questionnaire in detecting moderate-to-severe OSA was 77.6, $58.8,50.6$ and $82.9 \%$, respectively (Tan et al. 2017). We defined underweight as $\mathrm{BMI}<18.5 \mathrm{~kg} / \mathrm{m}^{2}$, normal: BMI $18.5-24.9 \mathrm{~kg} / \mathrm{m}^{2}$, overweight: BMI $25.0-29.9 \mathrm{~kg} / \mathrm{m}^{2}$ and obese: BMI $\geq 30.0 \mathrm{~kg} / \mathrm{m}^{2}$ (Centers for Disease Control and Prevention n.d.). Individuals who smoked at least 1 cigarette in the past 6 months were regarded as current smokers, those who last smoked over 6 months or self-reported quitting smoking were considered past smokers and those who never smoked were regarded 
as non-smokers. Alcohol drinking was defined as at least a once consumption every week. Hypertension was defined as SBP $\geq 140 \mathrm{mmHg}$ or DBP $\geq 90 \mathrm{mmHg}$, or use of blood pressure lowering agents (Chobanian et al. 2003). Diabetes was diagnosed using a random blood glucose $(\mathrm{RBG}) \geq 11.1 \mathrm{mmol} / \mathrm{L}$ and/or fasting blood glucose $(\mathrm{FBG}) \geq 7 \mathrm{mmol} / \mathrm{L}$ or use of glucose-lowering agents (American Diabetes Association 2015).

\section{Statistical analysis}

All statistical analyses utilized STATA v11.0 software. Summaries of continuous variables are presented as means $( \pm \mathrm{SD})$ and categorical variables are presented as frequencies (percentages). Pearson Chi square and Student's T-test were used in comparison of categorical and continuous variables respectively. Logistic regression analyses were used to assess for factors associated with OSA. Stepwise and forward selection procedure was used to add and assess the statistically significant variables in the multivariate regression model. The multivariate model was fitted with baseline covariates associated with OSA by bivariate analysis at the $<0.05$ significance level. Furthermore, subgroup analyses to determine the odds for OSA by hypertension status was performed and the findings are presented graphically in a forest plot. Odd ratios with $95 \%$ confidence intervals and $p$-values are reported. All tests were 2 -sided and $p<0.05$ was used to indicate a statistical significance.

\section{Results}

\section{Study population characteristics}

A total of 1974 individuals (i.e. 1289 hypertensive and 685 normotensives) were enrolled in this study. Table 1 displays the sociodemographic and clinical characteristics of study participants. The mean age was 53.4years and $63.8 \%$ of participants were aged 60 years or less. Females constituted the large majority $(60.4 \%)$ of participants. $54.0 \%$ of participants had attained primary school as their highest level of education and $67.9 \%$ were in marriage. Over $80 \%$ of participants dwelled in urban areas, $63.3 \%$ had a regular income generating activity and $26.4 \%$ worked at least $8 \mathrm{~h}$ a day. Over one-sixth (15.9\%) had a positive smoking history and $10 \%$ were current alcohol consumers. Nearly three quarters (74.1\%) of participants had excess body weight, $11.6 \%$ had diabetes, $8.0 \%$ had asthma and $18.6 \%$ had history of recurrent nasal congestion. Positive family history of snoring was reported by $43.1 \%$ of participants and $36.9 \%$ had a personal history of snoring. Surprisingly, none of the participants in this study was aware of OSA and its potential in causing or worsening CVDs and other chronic conditions.

\section{Prevalence and correlates of OSA}

Overall, 624 (31.6\%) of all participants were categorized as having high-risk for OSA. Persons with hypertension displayed a higher frequency (42.1\%) of suffering from OSA compared to their normotensive counterparts $(11.8 \%), p<0.001$. Furthermore, the prevalence of highrisk for OSA was similar among hypertensives regardless of their BP range i.e. $41.1 \%$ in SBP < 140, 43.2\% in SBP140159 and $42.4 \%$ in $\mathrm{SBP} \geq 160$. Moreover, individuals with hypertension had a $6.8 \%$ prevalence of EDS compared to $5.6 \%$ among controls, $p=0.3$, Table 2. During bivariate analyses, participants with high-risk for OSA were older (57.9 vs $51.3, p<0.001$ ) and a positive linear correlation between age and OSA until the age group 60-70 years was observed i.e. < 40 years, $13.4 \%$; $41-50$ years, $29.0 \%$; 51-60 years, $36.7 \%$; $61-70$ years, $42.9 \%$; and $>70$ years, $36.3 \%$. Moreover, participants with high-risk for OSA had a higher comorbidity history [hypertension $(87.0 \%$ vs $55.3 \%, p<0.001)$, diabetes $(18.3 \%$ vs $8.4 \%, p<0.001)$, stroke $(7.2 \%$ vs $5.0 \%, p=0.05)$ and recurrent nasal congestion $(25.8 \%$ vs $15.3 \%, p<0.001)]$, and a higher frequency of known risk factors i.e. positive smoking history ( $18.3 \%$ vs $14.7 \%, p=0.04)$, increased body weight $(88.9 \%$ vs $67.2 \%, p<0.001)$, increased neck circumference $(88.6 \%$ vs $72.3 \%, p<0.001)$, post-menopause $(83.5 \%$ vs $59.8 \%$, $p<0.001)$, positive family $(70.2 \%$ vs $30.6 \%, p<0.001)$ and personal history of snoring $(99.7 \%$ vs $7.9 \%, p<0.001)$, and a higher ESS score $(42.8 \%$ vs $27.6 \%, p<0.001)$; Table 3 . Males and females displayed similar odds of suffering from OSA (i.e. $31.7 \%$ vs $31.5 \%, p=0.9$ ).

In a logistic regression model of 16 characteristics, 7 factors i.e. hypertension (OR 5.1, 95\% CI 3.2-8.2, $p<0.001$ ), diabetes mellitus (OR 2.2, 95\% CI 1.3-3.5, $p<0.01$ ), chronic nasal congestion (OR 1.6, 95\% CI 1.1$2.5, p=0.01$ ), obesity (OR $2.4,95 \%$ CI $1.8-3.3, p<0.001$ ), increased neck circumference (OR 2.7, 95\% CI 1.2-6.4, $p=0.02$ ), family history of snoring (OR $5.5,95 \%$ CI 4.0 $7.5, p<0.001)$, and working $>8 \mathrm{~h} / 24 \mathrm{~h}$ (OR $0.6,95 \% \mathrm{CI}$ 0.4-1.0, $p=0.03$ ) were found to have an independent association of suffering from OSA, Table 4. Furthermore, as displayed in Fig. 1, participants with hypertension had superior odds for suffering from OSA compared to their normotensive counterparts across all subgroup analyses. For instance, among individuals with $\mathrm{BMI} \geq 25$ those with hypertension displayed a 4-fold chance of having a higher risk of suffering from OSA compared to normotensive persons, OR 4.0, 95\% CI 3.0-5.5, $p<0.001$. Similarly, among participants with a positive family history of snoring, those with hypertension had a 7-fold likelihood of having a higher risk of suffering from OSA compared to their counterparts with negative history of hypertension, OR 7.1, 95\%CI 5.1-10.1, $p<0.001$. Nevertheless, participants with diabetes displayed similar odds of 
Table 1 Sociodemographic characteristics of study participants by hypertension status

\begin{tabular}{|c|c|c|c|c|}
\hline Characteristic & $\begin{array}{l}\text { ALL } \\
N=1974\end{array}$ & $\begin{array}{l}\text { Hypertensive } \\
n=1289\end{array}$ & $\begin{array}{l}\text { Normotensive } \\
n=685\end{array}$ & $p$-value \\
\hline Age (Mean, SD) & $53.4(15.0)$ & $59.8(11.8)$ & $41.3(13.0)$ & $<0.001$ \\
\hline Range & $18-98$ & $18-98$ & $18-83$ & \\
\hline \multicolumn{5}{|l|}{ Age group } \\
\hline$>60$ & $714(36.2 \%)$ & $653(50.7 \%)$ & $61(08.9 \%)$ & \\
\hline$\leq 60$ & $1260(63.8 \%)$ & $636(49.3 \%)$ & 624 (91.1\%) & $<0.001$ \\
\hline \multicolumn{5}{|l|}{ Sex } \\
\hline Male & $782(39.6 \%)$ & $483(37.5 \%)$ & $299(43.7 \%)$ & \\
\hline Female & $1192(60.4 \%)$ & $806(62.5 \%)$ & $386(56.4 \%)$ & $<0.01$ \\
\hline \multicolumn{5}{|l|}{ Education } \\
\hline No Formal & $117(05.9 \%)$ & $100(07.8 \%)$ & $17(02.5 \%)$ & $<0.001$ \\
\hline Primary & $950(48.1 \%)$ & $643(49.9 \%)$ & $307(44.8 \%)$ & 0.03 \\
\hline Secondary & $513(26.0 \%)$ & $318(24.6 \%)$ & 195 (28.5\%) & 0.06 \\
\hline University & 394 (20.0\%) & $228(17.7 \%)$ & $166(24.2 \%)$ & $<0.001$ \\
\hline \multicolumn{5}{|l|}{ Marital status } \\
\hline Single & $192(09.7 \%)$ & 48 (03.7\%) & $144(21.0 \%)$ & $<0.001$ \\
\hline Married & $1341(67.9 \%)$ & $873(67.7 \%)$ & $468(68.3 \%)$ & 0.79 \\
\hline Divorced & $128(06.5 \%)$ & $82(06.4 \%)$ & $46(06.7 \%)$ & 0.80 \\
\hline Widowed & $313(15.9 \%)$ & $286(22.2 \%)$ & $27(04.0 \%)$ & $<0.001$ \\
\hline \multicolumn{5}{|l|}{ Occupation } \\
\hline Jobless & $371(18.8 \%)$ & $253(19.6 \%)$ & $118(17.2 \%)$ & 0.19 \\
\hline Self-employed & $888(45.0 \%)$ & $487(37.8 \%)$ & $401(58.5 \%)$ & $<0.001$ \\
\hline Employed & $362(18.3 \%)$ & $219(17.0 \%)$ & $143(20.9 \%)$ & 0.03 \\
\hline Retired & $353(17.9 \%)$ & $330(25.6 \%)$ & $23(03.4 \%)$ & $<0.001$ \\
\hline \multicolumn{5}{|l|}{ Work duration (hours) } \\
\hline Mean (SD) & $6.2(3.8)$ & $5.4(3.5)$ & $7.7(3.9)$ & $<0.001$ \\
\hline$>8 \mathrm{~h} /$ day & $521(26.4 \%)$ & $256(19.9 \%)$ & $265(38.7 \%)$ & $<0.001$ \\
\hline \multicolumn{5}{|l|}{ Residence } \\
\hline Urban & $1618(82.0 \%)$ & $1030(79.9 \%)$ & $588(85.8 \%)$ & \\
\hline Rural & $356(18.0 \%)$ & $259(20.1 \%)$ & $97(14.2 \%)$ & $<0.01$ \\
\hline \multicolumn{5}{|l|}{ Personal Disease History (\% Yes) } \\
\hline Diabetes & $228(11.6 \%)$ & $211(16.4 \%)$ & $17(02.5 \%)$ & $<0.001$ \\
\hline Asthma & $158(08.0 \%)$ & $107(08.3 \%)$ & $51(07.5 \%)$ & 0.53 \\
\hline Stroke & $113(05.7 \%)$ & $103(08.0 \%)$ & $10(01.5 \%)$ & $<0.001$ \\
\hline Recurrent nocturnal nasal congestion & $367(18.6 \%)$ & $270(21.0 \%)$ & $97(14.2 \%)$ & $<0.001$ \\
\hline \multicolumn{5}{|l|}{ Smoking status } \\
\hline Ever smoker & $313(15.9 \%)$ & $284(14.3 \%)$ & $129(18.8 \%)$ & \\
\hline Never & $1661(84.1 \%)$ & $1105(85.7 \%)$ & $556(81.2 \%)$ & $<0.01$ \\
\hline \multicolumn{5}{|l|}{ Alcohol intake (current) } \\
\hline Yes & $197(10.0 \%)$ & $70(05.4 \%)$ & $127(18.5 \%)$ & \\
\hline No & $1777(90.0 \%)$ & $1219(94.6 \%)$ & $558(81.5 \%)$ & $<0.001$ \\
\hline $\begin{array}{l}\text { Body Mass Index (mean, SD) } \\
\text { BMI categories }\end{array}$ & $29.1(6.2)$ & $30.3(6.3)$ & $26.9(5.5)$ & $<0.001$ \\
\hline Underweight & $34(01.7 \%)$ & $11(0.9 \%)$ & $23(03.4 \%)$ & $<0.001$ \\
\hline Normal & $478(24.2 \%)$ & $225(17.4 \%)$ & $253(36.9 \%)$ & $<0.001$ \\
\hline Overweight & $680(34.5 \%)$ & $447(34.7 \%)$ & $233(34.0 \%)$ & 0.76 \\
\hline Obese & $782(39.6 \%)$ & $606(47.0 \%)$ & $176(25.7 \%)$ & $<0.001$ \\
\hline Waist circumference (mean [cm], SD) & $98.1(14.0)$ & $101.0(13.4)$ & $92.8(13.4)$ & $<0.001$ \\
\hline Men $>90 \mathrm{~cm}$ & $486(62.1 \%)$ & $357(73.9 \%)$ & $129(43.1 \%)$ & $<0.001$ \\
\hline Women $>80 \mathrm{~cm}$ & $1089(91.4 \%)$ & $766(95.0 \%)$ & $323(83.7 \%)$ & $<0.001$ \\
\hline
\end{tabular}


Table 1 (continued)

\begin{tabular}{|c|c|c|c|c|}
\hline Characteristic & $\begin{array}{l}\text { ALL } \\
N=1974\end{array}$ & $\begin{array}{l}\text { Hypertensive } \\
n=1289\end{array}$ & $\begin{array}{l}\text { Normotensive } \\
n=685\end{array}$ & $p$-value \\
\hline Neck circumference (mean[cm], SD) & $36.8(3.5)$ & $37.2(3.4)$ & $35.9(3.6)$ & $<0.001$ \\
\hline Men $>37 \mathrm{~cm}$ & $434(55.5 \%)$ & $314(65.0 \%)$ & $120(40.1 \%)$ & $<0.001$ \\
\hline Women $>31 \mathrm{~cm}$ & 1095 (91.9\%) & $767(95.2 \%)$ & $328(85.0 \%)$ & $<0.001$ \\
\hline \multicolumn{5}{|l|}{ Family history of snoring } \\
\hline Positive & $851(43.1 \%)$ & $566(43.9 \%)$ & $285(41.6 \%)$ & 0.33 \\
\hline Negative & $982(49.8 \%)$ & $617(47.9 \%)$ & $365(53.3 \%)$ & 0.02 \\
\hline Don't Know & $141(07.1 \%)$ & $106(08.2 \%)$ & $35(05.1 \%)$ & 0.01 \\
\hline \multicolumn{5}{|l|}{ Personal history of snoring } \\
\hline Positive & $729(36.9 \%)$ & $564(43.8 \%)$ & $165(24.0 \%)$ & $<0.001$ \\
\hline Negative & $1156(58.6 \%)$ & $659(51.1 \%)$ & $497(72.6 \%)$ & $<0.001$ \\
\hline Don't Know & $89(04.5 \%)$ & $66(05.1 \%)$ & $23(03.4 \%)$ & 0.08 \\
\hline \multicolumn{5}{|l|}{ OSA awareness } \\
\hline Yes & $0(0 \%)$ & $0(0 \%)$ & $0(0 \%)$ & 1.0 \\
\hline No & $1974(100 \%)$ & $1289(100 \%)$ & $685(100 \%)$ & 1.0 \\
\hline
\end{tabular}

Table 2 Prevalence and pattern of Excessive Daytime Sleepiness by hypertension status

\begin{tabular}{llll}
\hline EDS & \multicolumn{2}{l}{ Frequency (\%) } & p-value \\
\cline { 2 - 3 } & Hypertensive & Normotensive & \\
\hline Unlikely & $635(49.3 \%)$ & $373(54.5 \%)$ & 0.03 \\
Average & $210(16.3 \%)$ & $116(16.9 \%)$ & 0.7 \\
Excessive situational & $356(27.6 \%)$ & $158(23.1 \%)$ & 0.03 \\
Excessively sleepy & $88(06.8 \%)$ & $38(05.6 \%)$ & 0.3 \\
\hline
\end{tabular}

suffering from OSA regardless of their hypertension status, OR 1.9, 95\%CI 0.6-6.6, $p=0.21$.

\section{Discussion}

Over the past five decades, OSA, a potentially treatable disorder that is characterized by cyclic intermittent hypoxia and the disruption of sleep architecture has been increasingly recognized as a widespread syndrome causing significant population health burden. Despite its ubiquity and potential to either cause or modify the course of other chronic disorders, OSA continues to be underdiagnosed and undertreated worldwide (Punjabi 2008; Young et al. 1997b). With over two-fifth of hypertensives having high-risk of suffering from OSA, this present study echoes findings from previous studies (Kario 2009; Lavie et al. 2000; Calhoun and Harding 2010; Börgel et al. 2004; Logan et al. 2001; Peppard et al. 2000a; Lavie and Hoffstein 2001; Bixler et al. 2000; Young et al. 1997a; Nieto et al. 2000; Marin et al. 2012; Pedrosa et al. 2011; Gonçalves et al. 2007; Hou et al. 2018; Sapiña-Beltrán et al. 2019; Patel et al.
2019; Ahmad et al. 2017; Belyavskiy et al. 2019; Tam et al. 2019; Steiropoulos et al. 2010; Zhang and Si 2012; Wolf and Narkiewicz 2017; Jelic et al. 2002; Minoguchi et al. 2005; Jehan et al. 2020; Fava et al. 2014; Epstein et al. 2009; Parati et al. 2014; Parati et al. 2012; Somers et al. 2008) that OSA is highly prevalent among hypertensive individuals. In unison with the literature (Hedner et al. 2006; Sia et al. 2017; Redline et al. 2014; Rezaie and Khaledi-Paveh 2018), this study has revealed critically low awareness regarding OSA and its associated health-related consequences. Amid the rapid rising obesity, hypertension and diabetes rates, it is pivotal to intensify the ongoing health education campaigns to increase awareness so that they coincide with the temporal trends in public knowledge of OSA amongst others.

Moreover, a positive linear correlation between age and OSA until the age group 60-70years was observed in this present study. Such findings are literally identical to the Sleep Heart Health Study which demonstrated a simple, positive linear correlation between age and OSA until the age of about 65 years (Young et al. 2002). Furthermore, this linear trend between OSA and age until about the age 65 then levelling off or even decreasing has been reported by a couple of other recent studies (Huang et al. 2018; Marshall et al. 2014; Fietze et al. 2019). We observed similar EDS rates among hypertensives and normotensives. Previous studies have revealed conflicting results with some (Goldstein et al. 2004; Mbatchou Ngahane et al. 2015) showing independent association between EDS and hypertension while others did not (Tam et al. 2019; Pefura-Yone et al. 2020; Martynowicz et al. 2017). As witnessed in this study, such 
Table 3 Sociodemographic and Clinical characteristics comparison by OSA status

\begin{tabular}{|c|c|c|c|}
\hline Characteristic & $\begin{array}{l}\text { High-risk OSA } \\
n=624\end{array}$ & $\begin{array}{l}\text { Low-risk OSA } \\
n=1350\end{array}$ & $p$-value \\
\hline Age (Mean, SD) & $57.9(12.3)$ & $51.3(15.7)$ & $<0.001$ \\
\hline \multicolumn{4}{|l|}{$\mathrm{n}$} \\
\hline $\begin{array}{l}<40 \text { years }^{c} \\
442\end{array}$ & $59(13.4 \%)$ & & - \\
\hline $\begin{array}{l}41-50 \\
338\end{array}$ & $98(29.0 \%)$ & & $<0.001$ \\
\hline $\begin{array}{l}51-60 \\
480\end{array}$ & $176(36.7 \%)$ & & $<0.001$ \\
\hline $\begin{array}{l}61-70 \\
480\end{array}$ & $206(42.9 \%)$ & & $<0.001$ \\
\hline $\begin{array}{l}>70 \text { years } \\
234\end{array}$ & $85(36.3 \%)$ & & $<0.001$ \\
\hline Female sex & $376(60.3 \%)$ & $816(60.4 \%)$ & 1.0 \\
\hline$\leq$ Primary education & $346(55.5 \%)$ & $721(53.4 \%)$ & 0.38 \\
\hline Married & $439(70.4 \%)$ & $902(66.8 \%)$ & 0.11 \\
\hline Work duration $>8 \mathrm{~h} /$ day & $135(21.6 \%)$ & $386(28.6 \%)$ & 0.001 \\
\hline Urban residency & $505(80.9 \%)$ & $1113(82.4 \%)$ & 0.42 \\
\hline \multicolumn{4}{|l|}{ Comorbidities history } \\
\hline Hypertension & $543(87.0 \%)$ & $746(55.3 \%)$ & $<0.001$ \\
\hline Diabetes & $114(18.3 \%)$ & $114(08.4 \%)$ & $<0.001$ \\
\hline Asthma & $48(07.7 \%)$ & $110(08.2 \%)$ & 0.70 \\
\hline Stroke & $45(07.2 \%)$ & $68(05.0 \%)$ & 0.05 \\
\hline Recurrent nocturnal nasal congestion & $161(25.8 \%)$ & $206(15.3 \%)$ & $<0.001$ \\
\hline Menopause $^{a}$ & $314(83.5 \%)$ & $488(59.8 \%)$ & $<0.001$ \\
\hline Ever smoker & $114(18.3 \%)$ & $199(14.7 \%)$ & 0.04 \\
\hline Current drinker & $43(06.9 \%)$ & $154(11.4 \%)$ & 0.002 \\
\hline \multicolumn{4}{|l|}{ Measures of obesity } \\
\hline $\mathrm{BMI} \geq 25$ & $555(88.9 \%)$ & $907(67.2 \%)$ & $<0.001$ \\
\hline Neck $>37 \mathrm{~cm} \mathrm{M/31} \mathrm{cm} \mathrm{F}$ & $553(88.6 \%)$ & $976(72.3 \%)$ & $<0.001$ \\
\hline Waist $>90 \mathrm{~cm} \mathrm{M} / 80 \mathrm{~cm} \mathrm{~F}$ & $572(91.7 \%)$ & $1003(74.3 \%)$ & $<0.001$ \\
\hline \multicolumn{4}{|l|}{ Snoring history } \\
\hline Positive family & $438(70.2 \%)$ & $413(30.6 \%)$ & $<0.001$ \\
\hline Positive personal & $622(99.7 \%)$ & $107(07.9 \%)$ & $<0.001$ \\
\hline$S B P \geq 140$ and/or $D B P \geq 90^{b}$ & $365(67.2 \%)$ & $482(64.6 \%)$ & 0.26 \\
\hline $\begin{array}{l}\mathrm{SBP}<140^{\mathrm{bc}} \\
504\end{array}$ & $207(41.1 \%)$ & & - \\
\hline $\begin{array}{l}\text { SBP } 140-159^{b} \\
424\end{array}$ & $183(43.2 \%)$ & & 0.51 \\
\hline $\begin{array}{l}S B P \geq 160^{b} \\
361\end{array}$ & $153(42.4 \%)$ & & 0.70 \\
\hline ESS score $\geq 10$ & $267(42.8 \%)$ & $373(27.6 \%)$ & $<0.001$ \\
\hline
\end{tabular}

${ }^{a}$ assessed in women, $n=1192 ;{ }^{b}$ assessed in hypertensives, $n=1289 ;{ }^{c}$ reference group

controversy can largely be explained by a high prevalence of overweight and obesity which independently increases the risk of EDS (Mokhber et al. 2016; Panossian and Veasey 2012; Hayley et al. 2014).

Nonetheless, with a 2-fold likelihood of suffering from OSA among obese individuals, our findings are in consonance with numerous other studies which have revealed excessive body weight as a potential factor for the development and progression of OSA (Young et al. 2002; Durán et al. 2001; Resta et al. 2001; Wolk and Somers 2006; Peppard et al. 2000b; Romero-Corral et al. 2010; Young et al. 1993; Bixler et al. 1998; Ip et al. 2004; Ip et al. 2001). Furthermore, as it was vivid in this study, other anthropometric measurements including neck and waist circumference 
Table 4 Logistic regression analyses for factors associated with OSA

\begin{tabular}{|c|c|c|c|c|c|c|c|}
\hline Characteristic & Comparative & OR & $95 \% \mathrm{Cl}$ & $p$-value & Adj.OR & $95 \% \mathrm{Cl}$ & $p$-value \\
\hline Age $>60$ & Age $\leq 60$ & 1.9 & $1.6-2.3$ & $<0.001$ & 1.2 & $0.8-1.7$ & 0.32 \\
\hline Male & Female & 1.0 & $0.8-1.2$ & 0.94 & - & - & - \\
\hline Diabetes & Diabetes-free & 2.4 & $1.8-3.2$ & $<0.001$ & 2.2 & $1.3-3.5$ & $<0.01$ \\
\hline Asthmatic & Asthma-free & 0.9 & $0.7-1.3$ & 0.73 & - & - & - \\
\hline History of stroke & Stroke-free & 1.5 & $1.0-2.2$ & 0.05 & 0.8 & $0.4-1.6$ & 0.57 \\
\hline Chronic nasal congestion & No congestion & 1.9 & $1.5-2.4$ & $<0.001$ & 1.6 & $1.1-2.5$ & 0.01 \\
\hline Ever smoker & Never smoker & 1.3 & $1.0-1.7$ & 0.05 & 0.8 & $0.4-1.8$ & 0.63 \\
\hline $\mathrm{BMI} \geq 30$ & $\mathrm{BMI}<30$ & 3.7 & $3.0-4.5$ & $<0.001$ & 2.4 & $1.8-3.3$ & $<0.001$ \\
\hline Neck $>37 \mathrm{~cm} \mathrm{M/31} \mathrm{cm} \mathrm{F}$ & Normal Neck & 3.0 & $2.3-3.9$ & $<0.001$ & 2.7 & $1.2-6.4$ & 0.02 \\
\hline Family history of snoring & Negative history & 6.1 & $4.9-7.6$ & $<0.001$ & 5.5 & $4.0-7.5$ & $<0.001$ \\
\hline Uncontrolled BP & $\mathrm{BP}<140 / 90$ & 0.9 & $0.7-1.1$ & 0.33 & - & - & - \\
\hline ESS score $\geq 10$ & ESS score $<10$ & 2.0 & $1.6-2.4$ & $<0.001$ & 1.3 & $0.9-1.8$ & 0.12 \\
\hline Hypertensive & Negative history & 5.4 & $4.2-7.0$ & $<0.001$ & 5.1 & $3.2-8.2$ & $<0.001$ \\
\hline Alcohol intake & Non drinker & 0.6 & $0.4-0.8$ & 0.002 & 1.4 & $0.7-3.1$ & 0.34 \\
\hline Post-menopausal & Pre-menopausal & 3.4 & $2.5-4.6$ & $<0.001$ & 1.2 & $0.8-1.9$ & 0.36 \\
\hline Works > 8h/day & works $\leq 8 \mathrm{~h} /$ day & 0.7 & $0.6-0.9$ & 0.001 & 0.6 & $0.4-1.0$ & 0.03 \\
\hline
\end{tabular}

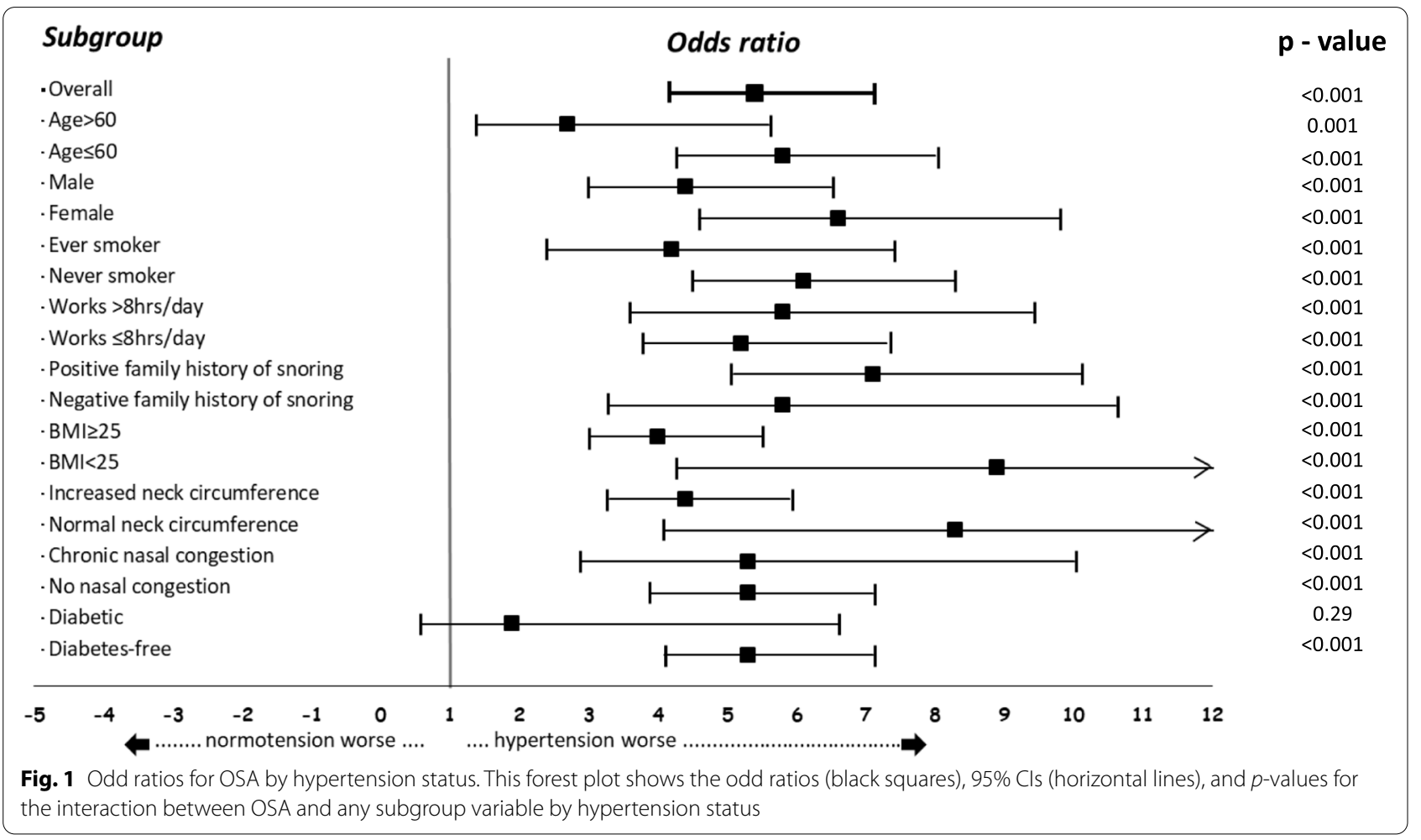

have revealed a monotonic relationship with OSA similar to previous studies (Young et al. 2002; Stradling and Crosby 1991; Ferini-Strambi et al. 1994; Davies et al. 1992; Grunstein et al. 1993). Moreover, it is postulated that each unit increase in BMI is associated with a $14 \%$ increased risk of developing OSA and the odds of moderate-severe
OSA increases 6-fold for every $10 \%$ weight gain (Peppard et al. 2000b). Conversely, a 10-15\% reduction in body weight has been shown to reduce OSA severity by $50 \%$ (Nousseir 2019). Our findings similar to majority of previous studies imply that excessive body weight is the strongest modifiable risk factor for OSA. 
Our association analysis furthermore disclosed a 2-fold increase in OSA likelihood among diabetes type 2 patients compared to their diabetes-free counterparts. Several cross-sectional and longitudinal studies have demonstrated an independent association between OSA and incident type 2 diabetes (Appleton et al. 2015; Kendzerska et al. 2014; Marshall et al. 2009; Nagayoshi et al. 2016; Botros et al. 2009; Celen et al. 2010; Lindberg et al. 2012) as well as with insulin resistance but diabetes-free persons (Punjabi et al. 2002; Punjabi and Beamer 2009; Iftikhar et al. 2015; Ip et al. 2002). Furthermore, a positive family history of snoring instigated an over 5 -fold increase in OSA likelihood and was found to be the strongest predictor of OSA in this study. Similarly, a Cleveland Family Study, the largest familial study investigating OSA revealed a near 2-fold risk among relatives of OSA compared to their control neighbors (Redline et al. 1995). Furthermore, studies involving monozygotic twins and siblings/children of persons with OSA have shown an up-to $50 \%$ increased odds of developing OSA compared to the general population (Ferini-Strambi et al. 1995; Friberg et al. 2009; Lundkvist et al. 2012; Szily et al. 2019). Additionally, numerous genetic studies (Palmer et al. 2003; Patel et al. 2007; Khalyfa et al. 2008; Gozal et al. 2007; Kalra et al. 2008; Farias Tempaku et al. 2020; Maierean et al. 2021; Kerz et al. 2021; Patel et al. 2012) have identified several novel genetic loci associated with a sleep apnea phenotype suggesting around two-fifth (Casale et al. 2009; Yue et al. 2008; Yue et al. 2005) of the OSA variance is attributable to genetic factors.

\section{Strengths and limitations}

The strengths of this study include (i) a sufficiently large sample to estimate the prevalence of OSA and conduct analyses stratified according to potential effect modifiers, (ii) the use of rigorous and standardized tools for data collection and utilization of qualified and competent personnel in all measurements, and (iii) presence of a comparative group in this study made the demonstration of outcome measure risk more robust. Nonetheless, this study is not short of limitations. The cross-sectional nature and convenience sampling method technique utilized in this study cannot preclude bias and limits both causality exploration and generalizability of findings. Prospective studies on the longitudinal association between OSA and hypertension incidence will help elucidate the true nature and magnitude of this intriguing association. Lastly, the gold standard for the diagnosis of OSA is an overnight polysomnography; however, because of its limited availability, complex technical support, and high cost, this study relied on a self-reported but validated assessment modality.

\section{Conclusion}

In conclusion; OSA, a largely modifiable CVD risk factor is considerably common among hypertensives in a tertiary health care setting in Tanzania. Positive family history of OSA was found to be the strongest associated factor; however, excess body weight proved to be the strongest modifiable risk factor. In view of its pervasiveness, OSA ought to be an integral part of the medical evaluation in hypertensive individuals. Furthermore, a multipronged approach to curb the escalating obesity epidemic is paramount in the battle against sleep-related breathing disorders.

\section{Abbreviations \\ 95\% Cl: 95\% Confidence Interval; BMI: Body mass index; BP: Blood pres- sure; CVD: Cardiovascular disease; DBP: Diastolic blood pressure; EDS: Excessive daytime sleepiness; FBG: Fasting blood glucose; JKCl: Jakaya Kikwete Cardiac Institute; OR: Odd Ratio; OSA: Obstructive sleep apnea; RBG: Random blood glucose; SSA: Sub Saharan Africa; SBP: Systolic blood pressure.}

\section{Acknowledgements}

We extend our gratitude to all participants for their willingness, tolerance and cooperation offered during this study.

\section{Authors' contributions}

PP and LRM conceived the study. MK, JM, NM, and HJS conducted all the interviews, as well as anthropometric and blood pressure measurements. ZM entered all the data. $\mathrm{HM}, \mathrm{SB}, \mathrm{SW}$, and $\mathrm{MJ}$ participated in patient management including counseling. PP performed all the data cleaning and analysis. The corresponding author (PP) wrote the first draft of the manuscript, and other authors contributed to and approved it. All authors made the decision to submit the manuscript for publication. All authors undertake responsibility for the accuracy and integrity of the analysis.

\section{Funding}

This study was sponsored by the PedPal Research Initiative.

\section{Availability of data and materials}

The datasets used and/or analyzed during the current study available from the corresponding author on reasonable request.

\section{Declarations}

Ethics approval and consent to participate

The study protocol was submitted to, and approved by the Ethical Committee, of the Jakaya Kikwete Cardiac Institute. Written informed consent was obtained from all study participants. This research was conducted in accordance with the Declaration of Helsinki.

\section{Consent for publication}

Not applicable.

\section{Competing interests}

The authors have no conflict of interest to declare.

\section{Author details}

${ }^{1}$ Unit of Research, Jakaya Kikwete Cardiac Institute, P.O Box 65141, Dar es Salaam, Tanzania. ${ }^{2}$ Cardiology, Jakaya Kikwete Cardiac Institute, P.O Box 65141, Dar es Salaam, Tanzania. ${ }^{3}$ Department of Psychiatry and Mental Health, Muhimbili University of Health and Allied Sciences, P.O Box 65001, Dar es Salaam, Tanzania. ${ }^{4}$ Nursing, Jakaya Kikwete Cardiac Institute, P.O Box 65141 Dar es Salaam, Tanzania. ${ }^{5}$ Clinical Support Services, Jakaya Kikwete Cardiac Institute, P.O Box 65141, Dar es Salaam, Tanzania. 
Received: 22 July 2021 Accepted: 13 October 2021 Published online: 15 December 2021

\section{References}

Ahmad M, Makati D, Akbar S. Review of and updates on hypertension in obstructive sleep apnea. Int J Hypertens. 2017;2017:1848375.

American Diabetes Association. Classification and diagnosis of diabetes. Sec 2. In Standards of Medical Care in Diabetes - 2015. Diabetes Care. 2015:38(Suppl. 1):S8-S16.

Appleton SL, Vakulin A, McEvoy RD. Nocturnal hypoxemia and severe obstructive sleep apnea are associated with incident type 2 diabetes in a population cohort of men. J Clin Sleep Med. 2015;11(6):609-14.

Belyavskiy E, Pieske-Kraigher E, Tadic M. Obstructive sleep apnea, hypertension, and obesity: a dangerous triad. J Clin Hypertens (Greenwich) 2019;21(10):1591-3.

Bixler EO, Vgontzas AN, Lin HM, et al. Association of hypertension and sleepdisordered breathing. Arch Intern Med. 2000;160:2289-95.

Bixler EO, Vgontzas AN, Ten Have T, Tyson K, Kales A. Effects of age on sleep apnea in men: I. prevalence and severity. Am J Respir Crit Care Med. 1998;157(1):144-8.

Börgel J, Sanner BM, Keskin F, et al. Obstructive sleep apnea and blood pressure. Interaction between the blood pressure-lowering effects of positive airway pressure therapy and antihypertensive drugs. Am J Hypertens. 2004;17(12 Pt 1):1081-7.

Botros N, Concato J, Mohsenin V. Obstructive sleep apnea as a risk factor for type 2 diabetes. Am J Med. 2009;122(12):1122-7.

Calhoun DA, Harding SM. Sleep and hypertension. Chest. 2010;138(2):434-43.

Casale M, Pappacena M, Rinaldi V, et al. Obstructive sleep apnea syndrome: from phenotype to genetic basis. Curr Genomics. 2009;10(2):119-26.

Celen YT, Hedner J, Carlson J. Impact of gender on incident diabetes mellitus in obstructive sleep apnea: a 16-year follow-up. J Clin Sleep Med. 2010;6(3):244-50.

Centers for Disease Control and Prevention. Division of Nutrition, Physical Activity, and Obesity. About Adult BMI. n.d. http://www.cdc.gov/healt hyweight/assessing/bmi/adult_bmi/.

Chobanian AV, Bakris GL, Black HR. Seventh report of the joint national committee on prevention, detection, evaluation, and treatment of high blood pressure. Hypertension. 2003;42:1206-52.

Davies RJO, Ali NJ, Stradling JR. Neck circumference and other clinical features in the diagnosis of the obstructive sleep apnea syndrome. Thorax. 1992:47:101-5.

Drakatos P, Ghiassi R, Jarrold I, Harris J, Abidi A, Douiri A, et al. The use of an online pictorial Epworth sleepiness scale in the assessment of age and gender specific differences in excessive daytime sleepiness. JThorac Dis. 2015:7(5):897-902

Durán J, Esnaola S, Rubio R, Iztueta A. Obstructive sleep apnea-hypopnea and related clinical features in a population-based sample of subjects aged 30 to 70 yr. Am J Respir Crit Care Med. 2001;163(3 Pt 1):685-9.

Durán-Cantolla J, Aizpuru F, Martínez-Null C, Barbé-Illa F. Obstructive sleep apnea_hypopnea and systemic hypertension. Sleep Med Rev. 2009;13(5):323-31.

Epstein MD, Kristo D, Strollo PJ, et al. Adult obstructive sleep apnea task force of the American Academy of sleep medicine. Clinical guideline for the evaluation, management and long-term care of obstructive sleep apnea in adults. J Clin Sleep Med. 2009;5:263-76.

Farias Tempaku P, Leite Santoro M, Bittencourt L, et al. Genome-wide association study reveals two novel risk alleles for incident obstructive sleep apnea in the EPISONO cohort. Sleep Med. 2020;66:24-32.

Fava C, Dorigoni S, Dalle Vedove F, et al. Effect of CPAP on blood pressure in patients with OSA/hypopnea a systematic review and meta-analysis. Chest. 2014;145:762-71.

Ferini-Strambi L, Calori G, Oldani A, et al. Snoring in twins. Respir Med. 1995:89(5):337-40.

Ferini-Strambi L, Zucconi M, Palazzi S, et al. Snoring and nocturnal oxygen desaturations in an Italian middle-aged male population: epidemiologic study with an ambulatory device. Chest. 1994;105:1759-64.

Fietze I, Laharnar N, Obst A, et al. Prevalence and association analysis of obstructive sleep apnea with gender and age differences - results of SHIP-trend. J Sleep Res. 2019:28(5):e12770.
Friberg D, Sundquist J, Li X, Hemminki K, Sundquist K. Sibling risk of pediatric obstructive sleep apnea syndrome and adenotonsillar hypertrophy. Sleep. 2009:32(8):1077-83.

Gami AS, Howard DE, Olson EJ, Somers VK. Day-night pattern of sudden death in obstructive sleep apnea. N Engl J Med. 2005:352(12):1206-14.

Goldstein IB, Ancoli-Israel S, Shapiro D. Relationship between daytime sleepiness and blood pressure in healthy older adults. Am J Hypertens. 2004;17(9):787-92.

Gonçalves SC, Martinez D, Gus M, et al. Obstructive sleep apnea and resistant hypertension: a case-control study. Chest. 2007;132:1858-62.

Gottlieb DJ, Yenokyan G, Newman AB, et al. Prospective study of obstructive sleep apnea and incident coronary heart disease and heart failure: the sleep heart health study. Circulation. 2010;122:352-60.

Gozal D, Capdevila OS, Kheirandish-Gozal L, et al. APOE epsilon 4 allele, cognitive dysfunction, and obstructive sleep apnea in children. Neurology. 2007;69:243-9.

Grunstein R, Wilcox I, Yang TS, Gould Y, Hedner J. Snoring and sleep apnea in men: association with central obesity and hypertension. Int J Obes. 1993;17:533-40.

Hayley AC, Williams $\sqcup$, Kennedy GA, et al. Excessive daytime sleepiness and body composition: a population-based study of adults. PLoS One. 2014;9(11):e112238.

Hedner J, Bengtsson-Bostrom K, Peker Y, et al. Hypertension prevalence in obstructive sleep apnoea and sex: a population-based case-control study. Eur Respir J. 2006;27:564-70.

Hou H, Zhao Y, Yu W, et al. Association of obstructive sleep apnea with hypertension: a systematic review and meta-analysis. J Glob Health. 2018;8(1):010405

Huang T, Lin BM, Markt SC, et al. Sex differences in the associations of obstructive sleep apnoea with epidemiological factors. Eur Respir J. 2018:51(3):1702421

Iftikhar IH, Hoyos CM, Phillips CL. Meta-analyses of the association of sleep apnea with insulin resistance, and the effects of CPAP on HOMA-IR, adiponectin, and visceral adipose fat. J Clin Sleep Med. 2015;11(4):475-85.

Ip MS, Lam B, Lauder IJ, et al. A community study of sleep-disordered breathing in middle-aged Chinese men in Hong Kong. Chest. 2001;119(1):62-9.

Ip MS, Lam B, Ng MM. Obstructive sleep apnea is independently associated with insulin resistance. Am J Respir Crit Care Med. 2002;165(5):670-6.

Ip MS, Lam B, Tang LC, Lauder IJ, Ip TY, Lam WK. A community study of sleepdisordered breathing in middle-aged Chinese women in Hong Kong: prevalence and gender differences. Chest. 2004;125(1):127-34.

Jehan S, Zizi F, Pandi-Perumal SR, et al. Obstructive sleep apnea, hypertension, resistant hypertension and cardiovascular disease. Sleep Med Disord. 2020;4(3):67-76

Jelic S, Bartels MN, Mateika JH, et al. Arterial stiffness increases during obstructive sleep apneas. Sleep. 2002;25(8):850-5.

Kalra M, Pal P, Kaushal R, et al. Association of ApoE genetic variants with obstructive sleep apnea in children. Sleep Med. 2008:3:260-5.

Kario K. Obstructive sleep apnea syndrome and hypertension: mechanism of the linkage and 24-h blood pressure control. Hypertens Res. 2009:32(7):537-41.

Kendzerska T, Gershon AS, Hawker G. Obstructive sleep apnea and incident diabetes. A historical cohort study. Am J Respir Crit Care Med. 2014;190(2):218-25

Kerz J, Schürmann P, Rothämel T, et al. Gene variants associated with obstructive sleep apnea (OSA) in relation to sudden infant death syndrome (SIDS). Int J Legal Med. 2021;135(4):1499-506.

Khalyfa A, Capdevila OS, Buazza MO, et al. Genome-wide gene expression profiling in children with non-obese obstructive sleep apnea. Sleep Med. 2008:10:75-86.

Kuniyoshi FH, Garcia-Touchard A, Gami AS, et al. Day-night variation of acute myocardial infarction in obstructive sleep apnea. J Am Coll Cardiol. 2008;52(5):343-6

Lattimore JD, Celermajer DS, Wilcox I. Obstructive sleep apnea and cardiovascular disease. J Am Coll Cardiol. 2003;41(9):1429-37.

Lavie $\mathrm{P}$, Herer $\mathrm{P}$, Hoffstein $\mathrm{V}$, et al. Obstructive sleep apnoea syndrome as a risk factor for hypertension: population study. BMJ. 2000;320(7233):479-82.

Lavie P, Hoffstein V. Sleep apnea syndrome: a possible contributing factor to resistant Sleep. 2001:24:721-5. 
Leung RS, Bradley TD. Sleep apnea and cardiovascular disease. Am J Respir Crit Care Med. 2001;164:2147-65.

Lindberg E, Theorell-Haglow J, Svensson M. Sleep apnea and glucose metabolism: a long-term follow-up in a community-based sample. Chest. 2012;142(4):935-42.

Logan AG, Perlikowski SM, Mente A, et al. High prevalence of unrecognized sleep apnoea in drug-resistant hypertension. J Hypertens. 2001;19:2271-7.

Lundkvist K, Sundquist K, Li X, Friberg D. Familial risk of sleep-disordered breathing. Sleep Med. 2012;13(6):668-73.

Maierean AD, Bordea IR, Salagean T, et al. Polymorphism of the serotonin transporter gene and the peripheral 5-Hydroxytryptamine in obstructive sleep apnea: what do we know and what are we looking for? A systematic review of the literature. Nat Sci Sleep. 2021;13:125-39.

Marin JM, Agustí A, Villar I, et al. Association between treated and untreated obstructive sleep apnea and risk of hypertension. JAMA. 2012;307:2169-76.

Marshall NS, Wong KK, Cullen SR, Knuiman MW, Grunstein RR. Sleep apnea and 20-year follow-up for all-cause mortality, stroke, and cancer incidence and mortality in the Busselton health study cohort. J Clin Sleep Med. 2014;10(4):355-62.

Marshall NS, Wong KK, Liu PY, et al. Sleep apnea as an independent risk factor for all-cause mortality: the Busselton health study. Sleep. 2008;31:1079-85.

Marshall NS, Wong KK, Phillips CL. Is sleep apnea an independent risk factor for prevalent and incident diabetes in the Busselton health study? J Clin Sleep Med. 2009;5(1):15-20.

Martynowicz H, Skomro R, Gać P, et al. The influence of hypertension on daytime sleepiness in obstructive sleep apnea. J Am Soc Hypertens. 2017;11(5):295-302

Mbatchou Ngahane BH, Nganda MM, Dzudie A, et al. Prevalence and determinants of excessive daytime sleepiness in hypertensive patients: a crosssectional study in Douala, Cameroon. BMJ Open. 2015;5:e008339.

Minoguchi K, Yokoe T, Tazaki T, et al. Increased carotid intima-media thickness and serum inflammatory markers in obstructive sleep apnea. Am J Respir Crit Care Med. 2005;172(5):625-30.

Mokhber S, Ravanbakhsh PZ, Jesmi F, et al. Comparing the excessive daytime sleepiness of obese and non-obese patients. Iran Red Crescent Med J. 2016;18(7):e21964.

Mooe T, Franklin KA, Wiklund U, Rabben T, Holmstrom K. Sleep-disordered breathing and myocardial ischemia in patients with coronary artery disease. Chest. 2000;117:1597-602.

Nagayoshi M, Punjabi NM, Selvin E. Obstructive sleep apnea and incident type 2 diabetes. Sleep Med. 2016;25:156-61.

Nieto FJ, Young TB, Lind BK, et al. Association of sleep-disordered breathing, sleep apnea, and hypertension in a large community-based study. Sleep Heart Health Study JAMA. 2000;283:1829-36.

Nousseir HM. Obesity: the major preventable risk factor of obstructive sleep apnea. J Curr Med Res Pract. 2019;4:1-5.

Palmer LJ, Buxbaum SG, Larkin EK, et al. A whole-genome scan for obstructive sleep apnea and obesity. Am J Hum Genet. 2003;72:340-50.

Panossian LA, Veasey SC. Daytime sleepiness in obesity: mechanisms beyond obstructive sleep apnea-a review. SLEEP. 2012;35(5):605-15.

Parati G, Lombardi C, Hedner J, et al. Position paper on the management of patients with obstructive sleep apnea and hypertension: joint recommendations by the European Society of Hypertension, by the European Respiratory Society and by the members of European COST (cooperation in scientific and technological research) ACTION B26 on obstructive sleep apnea. J Hypertension. 2012;30:633-46.

Parati G, Lombardi C, Hedner J, et al. Recommendations for the management of patients with obstructive sleep apnoea and hypertension. Eur Respir J. 2013;41:523-38.

Parati G, Ochoa JE, Bilo G, et al. Obstructive sleep apnea syndrome as a cause of resistant hypertension. Hypertens Res. 2014;37:601-13.

Patel AR, Patel AR, Singh S, Singh S, Khawaja I. The Association of Obstructive Sleep Apnea and Hypertension. Cureus. 2019;11(6):e4858.

Patel SR, Goodloe R, De G, et al. Association of Genetic Loci with sleep apnea in European Americans and African-Americans: the candidate Gene Association resource (CARe). PLoS One. 2012;7(11):e48836.
Patel SR, Larkin EK, Mignot E, et al. The association of angiotensin converting enzyme (ACE) polymorphisms with sleep apnea and hypertension. Sleep. 2007;30:531-3.

Pedrosa RP, Drager LF, Gonzaga CC, et al. Obstructive sleep apnea: the most common secondary cause of hypertension associated with resistant hypertension. Hypertension. 2011;58:811-7.

Pefura-Yone E, Kamga OJ, Balkissou AD, et al. Excessive daytime sleepiness and hypertension in Cameroonian adult population. Am J Intern Med. 2020:8(1):8-13.

Peker Y, Kraiczi H, Hedner J, Loth S, Johansson A, Bende M. An independent association between obstructive sleep apnoea and coronary artery disease. Eur Respir J. 1999;14:179-84.

Peppard PE, Young T, Palta M, Dempsey J, Skatrud J. Longitudinal study of moderate weight change and sleep-disordered breathing. JAMA. 2000b;284(23):3015-21.

Peppard PE, Young T, Palta M, Skatrud J. Prospective study of the association between sleep-disordered breathing and hypertension. N Engl J Med. 2000a:342:1378-84

Pinto JA, Ribeiro DK, Cavallini AF, et al. Comorbidities associated with obstructive sleep apnea: a retrospective study. Int Arch Otorhinolaryngol. 2016;20(2):145-50.

Punjabi NM. The epidemiology of adult obstructive sleep apnea. Proc Am Thorac Soc. 2008;5(2):136-43.

Punjabi NM, Beamer BA. Alterations in glucose disposal in sleep-disordered breathing. Am J Respir Crit Care Med. 2009;179(3):235-40.

Punjabi NM, Caffo BS, Goodwin JL, et al. Sleep-disordered breathing and mortality: a prospective cohort study. PLoS Med. 2009:6:e1000132.

Punjabi NM, Sorkin JD, Katzel LI. Sleep-disordered breathing and insulin resistance in middle-aged and overweight men. Am J Respir Crit Care Med. 2002;165(5):677-82.

Redline S, Sotres-Alvarez D, Loredo J, et al. Sleep-disordered breathing in Hispanic/Latino individuals of diverse backgrounds. The Hispanic community health study/study of Latinos. Am J Respir Crit Care Med. 2014;189(3):335-44.

Redline S, Tishler PV, Tosteson TD, et al. The familial aggregation of obstructive sleep apnea. Am J Respir Crit Care Med. 1995;151(3 Pt 1):682-7.

Redline S, Yenokyan G, Gottlieb DJ, et al. Obstructive sleep apnea-hypopnea and incident stroke: the sleep heart health study. Am J Respir Crit Care Med. 2010;182:269-77.

Resta O, Foschino-Barbaro MP, Legari G, et al. Sleep-related breathing disorders, loud snoring and excessive daytime sleepiness in obese subjects. Int J Obes Relat Metab Disord. 2001;25(5):669-75.

Rezaie L, Khaledi-Paveh B. The knowledge and awareness of the general public about obstructive sleep apnea. J Kermanshah Univ Med Sci. 2018;22(2):e74025.

Romero-Corral A, Caples SM, Lopez-Jimenez F, Somers VK. Interactions between obesity and obstructive sleep apnea. Chest. 2010;137(3):711-9.

Sapiña-Beltrán E, Torres G, Benitez I, et al. Prevalence, characteristics, and Association of Obstructive Sleep Apnea with blood pressure control in patients with resistant hypertension. Ann Am Thorac Soc. 2019;16(11):1414-21.

Shah NA, Yaggi HK, Concato J, Mohsenin V. Obstructive sleep apnea as a risk factor for coronary events or cardiovascular death. Sleep Breathing. 2010;14(2):131-6.

Shahar E, Whitney CW, Redline S, et al. Sleep disordered breathing and cardiovascular disease: cross-sectional results of the sleep heart health study. Am J Respir Crit Care Med. 2001;163:19-25.

Sia CH, Hong Y, Tan LWL, et al. Awareness and knowledge of obstructive sleep apnea among the general population. Sleep Med. 2017;36:10-7.

Somers VK, White DP, Amin R, et al. Sleep apnea and cardiovascular disease: an AHA/ACC Foundation scientific statement from the AHA Council for high blood pressure research professional education committee, council on clinical cardiology, stroke council, and council on cardiovascular nursing. J Am Coll Cardiol. 2008;52:686-717.

Steiropoulos P, Papanas N, Nena E, et al. Inflammatory markers in middle-aged obese subjects: does obstructive sleep apnea syndrome play a role? Mediat Inflamm. 2010;2010:675320.

Stradling JR, Crosby JH. Predictors and prevalence of obstructive sleep apnea and snoring in 1001 middle aged men. Thorax. 1991:46:85-90. 
Szily M, Tarnoki AD, Tarnoki DL, et al. Genetic influences on the onset of obstructive sleep apnoea and daytime sleepiness: a twin study. Respir Res. 2019;20:125.

Tam W, Ng SS, To KW, Ko FW, Hui DS. The interaction between hypertension and obstructive sleep apnea on subjective daytime sleepiness. J Clin Hypertens (Greenwich). 2019;21(3):390-6.

Tan A, Yin JD, Tan LW, van Dam RM, Cheung YY, Lee CH. Using the Berlin questionnaire to predict obstructive sleep apnea in the general population. J Clin Sleep Med. 2017;13(3):427-32.

Wolf J, Narkiewicz K. Optimizing the management of uncontrolled/ resistant hypertension. The importance of sleep apnoea syndrome. Curr Vasc Pharmacol. 2017;16(1):44-53.

Wolk R, Somers VK. Obesity-related cardiovascular disease: implications of obstructive sleep apnea. Diabetes Obes Metab. 2006:8(3):250-60.

Worsnop CJ, Naughton MT, Barter CE, et al. The prevalence of obstructive sleep apnea in hypertensives. Am J Respir Crit Care Med. 1998;157(1):111-5.

Yaggi HK, Concato J, Kernan WN, et al. Obstructive sleep apnea as a risk factor for stroke and death. N Engl J Med. 2005;353:2034-41.

Young T, Evans L, Finn L, Palta M. Estimation of the clinically diagnosed proportion of sleep apnea syndrome in middle-aged men and women. Sleep. 1997b;20(9):705-6.

Young T, Finn L, Peppard PE, et al. Sleep disordered breathing and mortality: eighteen-year follow-up of the Wisconsin sleep cohort. Sleep. 2008;31:1071-8.
Young T, Palta M, Dempsey J, Skatrud J, Weber S, Badr S. The occurrence of sleep-disordered breathing among middle-aged adults. N Engl J Med. 1993:328(17):1230-5.

Young T, Peppard P, Palta M, et al. Population-based study of sleep-disordered breathing as a risk factor for hypertension. Arch Intern Med. 1997a;157:1746-52.

Young T, Shahar E, Nieto FJ, et al. Predictors of sleep-disordered breathing in community-dwelling adults: the sleep heart health study. Arch Intern Med. 2002;162(8):893-900.

Yue W, Liu H, Zhang J, et al. Association study of serotonin transporter gene polymorphisms with obstructive sleep apnea syndrome in Chinese Han population. Sleep. 2008;31(11):1535-41.

Yue W, Liu P, Hao W, et al. Association study of sleep apnea syndrome and polymorphisms in the serotonin transporter gene. Chin J Med Genet. 2005;22(5):533-6.

Zhang W, Si LY. Obstructive sleep apnea syndrome (OSAS) and hypertension: pathogenic mechanisms and possible therapeutic approaches. Ups J Med Sci. 2012;117(4):370-82.

\section{Publisher's Note}

Springer Nature remains neutral with regard to jurisdictional claims in published maps and institutional affiliations.
Ready to submit your research? Choose BMC and benefit from:

- fast, convenient online submission

- thorough peer review by experienced researchers in your field

- rapid publication on acceptance

- support for research data, including large and complex data types

- gold Open Access which fosters wider collaboration and increased citations

- maximum visibility for your research: over $100 \mathrm{M}$ website views per year

At BMC, research is always in progress.

Learn more biomedcentral.com/submissions 